\title{
Esprits mobiles, figures ancrées
}

Étudier les parcours migratoires des individus et leur rapport au sacré Mobile Spirits, Anchored Figures. Studying Individuals Migratory Itineraries and their Relationship to Sacred

\section{Delphine Burguet et Olivia Legrip-Randriambelo}

\section{OpenEdition Journals}

Édition électronique

URL : https://journals.openedition.org/etudesafricaines/33216

DOI : 10.4000/etudesafricaines.33216

ISSN : $1777-5353$

Éditeur

Éditions de l'EHESS

\section{Édition imprimée}

Date de publication : 15 mars 2021

Pagination : 105-113

ISBN : 9782713228766

ISSN : 0008-0055

Référence électronique

Delphine Burguet et Olivia Legrip-Randriambelo, « Esprits mobiles, figures ancrées », Cahiers d'études africaines [En ligne], 241 | 2021, mis en ligne le 15 mars 2021, consulté le 23 avril 2022. URL : http:// journals.openedition.org/etudesafricaines/33216; DOI : https://doi.org/10.4000/etudesafricaines. 33216 


\title{
Delphine Burguet \& Olivia Legrip-Randriambelo
}

\section{Esprits mobiles, figures ancrées}

\author{
Étudier les parcours migratoires des individus \\ et leur rapport au sacré
}

Ancêtres, pasteurs et rois: esprits réinventés et réseaux étendus

Ce dossier thématique étudie les circulations et les migrations au prisme des imaginaires du religieux dans un contexte contemporain où l'on observe une diversification des pratiques cultuelles en circulation et une redéfinition des liens sociaux en rapport au sacré (Levitt 2007). Interroger les continuités et les transformations en cours des identités religieuses et des imaginaires en contexte migratoire, a permis de révéler comment le religieux, les objets de croyances et les pratiques cultuelles constituent l'une des caractéristiques persistantes que les descendants de migrants conservent de leurs ancêtres (Bauman 2002). Les entités de «l'autre monde » peuvent se détacher de leur lieu d'ancrage dans le territoire géographique (Zempléni 1996: 339) : les esprits migrent, déménagent, s'exportent et se regroupent en des lieux saints cosmopolites (Burguet 2014).

De ce fait, le sacré n'est pas inamovible quand l'on parle de migration. L'ordre cosmologique est recréé et le rapport au sacré s'inscrit dans des objets spatiaux réaménagés ou nouvellement investis comme territoires. Ainsi, la Vierge de Santa Cruz déplacée d'Oran à Nîmes par les Européens d'Algérie devient la « Vierge des migrants » ou la « Vierge des exilés », et son sanctuaire s'inscrit dans le « diocèse de la dispersion » fondé dans le sud de la France (Baussant 2007); ou encore, dans le contexte des migrations capverdiennes, des « esprits sans patrie » conditionnent une « religion du voyage » (Laurent \& Plaideau 2010). Il en est de même pour la figure de saint Simon, sollicité par les Guatémaltèques candidats à la migration vers les États-Unis. Saint Simon est présenté comme maya sur les rives du lac Atitlan (alors appelé 
Maximón), puis comme un métis dans les grandes villes du pays et, enfin, comme un médecin italien migrant au Guatemala (Pédron-Colombani 2010).

L'expérience de la migration vécue par les esprits enclenche et rassure la démarche des croyants qui les sollicitent. Les études de cas présentées dans ce dossier traitent de la mobilité des esprits dans plusieurs régions d'Afrique, voire dans les Afriques, le phénomène dépassant le continent africain. On peut retenir comme particulièrement révélateur, à ce propos, le cas des esprits africains ayant migré vers les États-Unis. Ils sont légion chez les adeptes du mouvement akan, lesquels communiquent avec des esprits du Ghana ou du Togo réduits en esclavage et narrent leur histoire par la transe de possession (Guedj 2009). Le recours à ces esprits étrangers et locaux, mais dans tous les cas déterritorialisés, sont utilisés différemment en fonction des demandes des fidèles et permettent de répondre à un grand nombre de sollicitations. Il est alors question d'atteindre, à travers des esprits réinventés, un territoire symbolique et imaginé. Autrement dit, la religiosité s'insère dans une représentation fantasmée de la migration, par des esprits imprégnés d'un processus de migration.

Cette construction des imaginaires religieux se fait, en outre, par la recherche généalogique et des origines lorsque les populations ont migré, comme c'est le cas par exemple des Réunionnais vers Madagascar, des Africains Américains vers le Ghana (Guedj 2011) et l'île de Gorée au Sénégal (Mourre 2020), ou encore des Brésiliens adeptes des cultes afro-brésiliens vers « une Afrique mythifiée » (Capone 1999; Tall 2002). Ces contacts transnationaux ont permis de déployer, par le biais d'agences de voyage, des dynamiques religieuses, parfois folklorisées, appropriées, réinventées ou dépassées (Guedj 2011 : 190) à l'aune du retour au plus près des ancêtres ; ou encore par les festivals comme l'Odunde Festival qui a lieu sur la côte est des États-Unis et qui réunit les fidèles des religions dites « africaines », lesquelles regroupent, entre autres, les cultes yoruba, akan et kemet (ibid. : 210).

À partir de différentes échelles - locales, nationales, transnationales entre les pays du Sud ou des pays africains, les circulations impliquent l'investissement de lieux sacrés existants ou l'établissement de lieux nouveaux, ainsi que la reconfiguration des pratiques religieuses à chaque étape. Ainsi, les esprits et les dieux peuvent être considérés à la fois comme des facilitateurs de la migration et comme les motifs d'un lien social maintenu entre plusieurs espaces vécus. Ces esprits ou figures religieuses (saints, esprits royaux, djinns...) peuvent être également personnifiés, à travers des récits biographiques, où se construit une relation de proximité entre individus migrants et esprits migrants. Loin des analyses macropolitiques qui permettent de dégager les grandes tendances et les enjeux des flux migratoires, 
les microanalyses sont privilégiées afin de saisir les processus religieux en mouvement, les récits de vie, les imaginaires des croyants et les pratiques sociales des cultes, tout en prenant en compte les échelles locales des espaces sociaux et leurs recompositions.

Notre approche s'inspire des très nombreux travaux sur l'articulation entre religion et mobilité ${ }^{1}$ mais privilégie l'étude des acteurs religieux (pasteurs ou officiants de cultes aux esprits) et des entités surnaturelles. Les auteures du dossier s'attachent alors à ethnographier la quotidienneté et les recours individuels des croyants. Ainsi, les saints, esprits et autres figures du monde invisible s'adaptent aux nouvelles exigences et défis contemporains et les réseaux confessionnels accueillent de nouveaux personnages plus à même de traiter des demandes dans des contextes de migration nationale et/ou transnationale. Le dossier propose ainsi une lecture des façons de déplacer le sacré aux niveaux collectif ou individuel, voire intime, et offre un point de vue sur des communautés imaginées et des mouvements religieux à la fois visibles et invisibles.

Les contributions à ce dossier mettent en lumière les liens, les interactions et les formes d'adaptations rituelles dans les processus d'imbrication entre religion et migration. Les articles, rédigés par des anthropologues, font la part belle aux acteurs du religieux pour leur rôle moteur non seulement dans la transformation des pratiques cultuelles mais aussi dans la dynamique migratoire elle-même. Les discours de ces acteurs rapportés au fil des textes imputent la plasticité du religieux aux facultés d'adaptation des entités surnaturelles.

Ce dossier s'articule autour de quatre articles qui se répondent deux à deux. Les deux premiers textes, celui d'Élisabeth Rossé ainsi que celui de Delphine Burguet et Olivia Legrip-Randriambelo, portent sur la sous-région de l'océan Indien occidental à partir de terrains malgaches. Les textes de Carla Bertin et de Sophie Bava interrogent les engagements des acteurs évangéliques en migration au Bénin et au Maroc.

Outre leurs rapprochements thématiques et/ou régionaux évidents, ces travaux proposent des réflexions à plusieurs échelles : des mobilités humaines et surnaturelles localisées dans des pays ou des régions (Élisabeth Rossé et Carla Bertin) puis des processus similaires se jouant à des niveaux plus larges, voire intercontinentaux (Delphine Burguet \& Olivia Legrip-Randriambelo et Sophie Bava).

1. Voir notamment Meintel \& LeBlanc (2003), CApone (2004, 2010), Fourchard, Mary \& Otayek (2005), Fancello \& Mary (2010), Prencipe (2012), Dejean \& Endelstein (2013), Trouillet \& Lasseur (2016), CAPone \& Salzbrunn (2018). 
Réciprocité des enjeux du sacré et du politique

Les articles d'Élisabeth Rossé et de Delphine Burguet et Olivia LegripRandriambelo mettent en lumière la circulation des mondes humain et surnaturel entre les îles de l'océan Indien occidental (Beaujard 2012 ; Berger \& Blanchy 2014) ainsi que les liens politiques et économiques créés du fait de la circulation des esprits invoqués. Des lignes de perspective tirant vers l'histoire des sciences renforcent la profondeur d'analyse des données ethnographiques. Ces deux articles poursuivent ainsi un dialogue ancien et fructueux entre histoire et anthropologie dans l'océan Indien et participent à combler un manque de recherches sur les effets culturels des migrations à Madagascar.

Élisabeth Rossé montre bien, en s'appuyant sur les travaux de Michael Lambek (2002), comment, dans les rituels faisant appel à la transe de possession, des esprits appartenant à des périodes différentes de l'histoire malgache peuvent être convoqués simultanément par les devins-guérisseurs. Suivant la même idée, Delphine Burguet et Olivia Legrip-Randriambelo révèlent, à travers l'ethnographie du réseau d'esprits convoqués par une devineresseguérisseuse, comment des entités présentées comme des esprits internationaux sont néanmoins connectées à l'histoire de Madagascar ${ }^{2}$. Ici, les imaginaires religieux rencontrent un imaginaire national créé sur la base d'une (ré)invention de l'histoire de l'île.

Élisabeth Rossé étudie les processus de hiérarchisation entre les esprits autochtones et allochtones chez les populations tandroy migrantes de Madagascar. Après avoir exposé le contexte historique et anthropologique de la transformation des pratiques rituelles liées à l'esprit kokolampo, caractérisée par une fragilisation puis par une reprise d'autorité, l'auteure analyse les rapports entre kokolampo et tromba, qui alimentent un processus d'identification nationale à l'échelle de l'île et renforcent les structures lignagères locales. Les migrations des Tandroy ont modifié les pratiques du culte aux esprits et du culte aux ancêtres, donnant lieu à des tensions dans les imaginaires religieux : les esprits du pays tandroy, kokolampo, sont confrontés dans la migration à de nouveaux esprits sakalava, appelés tromba, ce qui permet à l'auteure de dépasser l'opposition classique autochtone/étranger. Ce procédé donne lieu à des identifications symboliques et nationales des esprits. En effet, les migrations tandroy au niveau national ont bouleversé le répertoire des esprits invoqués. Les kokolampo représentent un Madagascar étatique et les traditions ancestrales autochtones, tandis que les tromba symbolisent davantage le monde malgache précolonial associé à l'idée de modernité.

2. Sur les liens avec les esprits royaux, voir LEGRIP-RANDRIAMBELo (2016). 
Ici intervient un phénomène de réciprocité asymétrique, montrant un renforcement mutuel d'entités antagonistes jouant sur l'expression de l'identité collective, à la fois tandroy et malgache, locale et nationale.

Dans une approche comparative, Delphine Burguet et Olivia LegripRandriambelo analysent les enjeux contemporains de circulations transrégionales et transhistoriques des pratiques des cultes aux ancêtres à Madagascar. Ce questionnement s'inscrit dans le prolongement des études sur les mobilités des imaginaires religieux ancrés de longue date dans l'océan Indien occidental. En effet, les anthropologues ont montré les enjeux sociopolitiques des systèmes d'intégration progressifs d'esprits allochtones au répertoire d'esprits autochtones. Les entités de la surnature ou les esprits ancestraux de la Grande Île montrent la complexité politique des circulations des imaginaires religieux. Les auteures abordent ces enjeux politiques sous l'angle des migrations et de la mobilité des communautés, formant alors des réseaux cultuels interrégionaux et supralocaux. À partir de deux ethnographies situées sur les Hautes Terres, dans l'Imerina et dans le Betsileo, l'analyse éclaire la gestion religiopolitique et l'historicité des esprits invoqués par des devins-guérisseurs. Il s'agit de comprendre le pouvoir prétendu des esprits et l'autorité qu'ils exercent les uns sur les autres en fonction de leur identité, leur provenance, leur spécialité.

Les esprits ancrés et voyageurs mettent en scène la mobilité du religieux et reflètent les flux de migration, ce qui les amène à privilégier les connexions religieuses, culturelles et identitaires. De cette manière, sont saisis les enjeux des expériences migratoires qui questionnent les concepts de l'étranger et de l'étrange. Cette pluralité vise à montrer la capacité des acteurs religieux à capter du pouvoir divin (hasina) d'où qu'il vienne. Selon un mécanisme qui associe une hiérarchisation de ce répertoire, le culte aux ancêtres s'empare d'enjeux contemporains et propose une relecture de l'histoire de l'île. Ainsi, certains esprits exercent une autorité sur les autres : les esprits autochtones dominent les esprits allochtones, figures historiques des anciens oppresseurs de l'île. Cette domination est rendue visible par les modalités rituelles qui valorisent un ordre de préséance, des formules respectueuses, une matérialité des esprits dominants, mais cette domination est également rendue intelligible par les discours produits sur ces figures ancestrales.

\section{Évangélisation et ancrage des Églises en migration}

Les articles de Carla Bertin et de Sophie Bava présentent des pasteurs protestants engagés dans des parcours migratoires dont l'engagement 
religieux n'est pas nécessairement l'événement déclencheur de la migration. De l'intracontinental au national, les kilomètres parcourus (à plus ou moins grande distance) ont jalonné les expériences religieuses et façonné l'engagement dans les communautés. L'évangélisation en migration participe non seulement de processus d'ancrage dans un nouvel espace mais aussi de réancrage lors du retour des «planteurs d'église» dans leur village natal.

Carla Bertin étudie le parcours de "planteurs d'église » installés en zone urbaine et qui reviennent dans leur village natal, ainsi porteurs d'un imaginaire urbain. Ces acteurs sont fondamentaux dans l'implantation de nouveaux lieux de culte et incarnent à la fois la mobilité et l'ancrage. En édifiant de nouvelles Églises, ils participent à la transformation du paysage religieux. Ces « enfants du village », qui étaient partis en ville, reviennent au village afin d'investir les terres et les bâtiments familiaux pour les transformer en Église et en assemblées ouvertes à l'ensemble de la communauté villageoise à convertir. Il s'agit ici de saisir l'expérience migratoire comme le lieu d'apparition de la volonté du migrant d'opérer un repli sur le local dans le but d'entreprendre une conversion de son village de naissance, essentiellement par le biais du réseau familial. Les mobilités de retour au village d'origine sont les premières expériences d'évangélisation pour ces jeunes pasteurs, la conversion des plus proches étant estimée plus aisée. Par la suite, ils sont envoyés en mission pour de «bonnes mobilités » vers des espaces méconnus, cette fois.

Sophie Bava aborde la place et les rôles des pasteurs protestants au Maroc, à travers la notion de « théologie contextuelle » qui se déploie en trois temps au fil des parcours migratoires, passant d'une « théologie du passage », à une « théologie de l'ancrage » et enfin à une « théologie de l'installation ». À partir des parcours de trois migrants en provenance de la République démocratique du Congo et de Madagascar vers le Maroc, l'auteure propose de définir une théologie qui symbolise les différents registres de la pensée et de la pratique religieuses sollicités dans des expériences de mobilités. Notons que l'étude de la migration et de l'engagement religieux d'une Malgache vers le Maroc, mise en perspective avec les deux articles du premier ensemble consacré à la Grande Île, vient enrichir l'approche comparative et transversale de ce dossier. L'auteure s'attache également à analyser, sur cette « scène religieuse en mouvement», les références faites aux mobilités dans les prêches d'une « théologie de l'accompagnement » qui oriente les croyants dans les décisions qu'ils ont à prendre concernant leur parcours migratoire (rester ou quitter le Maroc, prendre la mer vers l'Europe, etc.).

Sophie Bava et Carla Bertin montrent enfin que les récits bibliques de migration conjugués à l'imaginaire des récits de vie des pasteurs historiques sont, à l'instar des esprits malgaches, reconstruits, enrichis, pour évoquer 
les héros des grandes épopées africaines. C'est par de tels procédés narratifs que les pasteurs du Bénin et du Maroc, tout comme les devins-guérisseurs et possédés malgaches, maîtrisent leur rôle social de spécialistes du religieux ayant surmonté des épreuves, parfois imaginaires elles aussi, et parviennent à asseoir leur légitimité dans des contextes parfois hostiles à leurs entreprises religieuses.

Institut des mondes africains (IMAF), EHESS, Aubervilliers ; LabEx COMOD, Université de Lyon.

\section{BIBLIOGRAPHIE}

Bauman M., 2002, « Migrant Settlement, Religion and Phase of Diaspora », A European Journal of International Migration and Ethnic Relations, 33-34 : 93-117.

Baussant M., 2007, «Penser les mémoires », Ethnologie française, 37 (3) : 389-394.

BaVA S. (DIR.), 2018, Dieu, les migrants et l'Afrique, Paris, L'Harmattan.

BAVA S. \& BoISSEVAIN K. (DIR.), 2014, dossier thématique « Routes migratoires africaines et dynamiques religieuses. Quels enjeux sociaux ?», L'année du Maghreb, 11.

BAVA S. \& CAPONE S., 2010, «Religions transnationales et migrations : regards croisés sur un champ en mouvement», Autrepart, 56 (4) : 3-15.

BAVA S. \& PICARD J., 2014, «La migration, moment de mobilité religieuse ? Le cas des Africains au Caire », Cahiers d'études du religieux. Recherches interdisciplinaires, $<$ http://journals.openedition.org/cerri/1390>, <DOI : 10.4000/cerri.1390>.

Beaujard P., 2012, Les mondes de l'océan Indien. Vol. 1 : De la formation de l'État au premier système-monde afro-eurasien (4e millénaire av. J.-C.-6e siècle apr. J.-C.). Vol. 2 : L'océan Indien au cœur des globalisations de l'Ancien Monde, Paris, Armand Colin.

Berger L. \& Blanchy S., 2014, «La fabrique des mondes insulaires. Altérités, inégalités et mobilités au sud-ouest de l'océan Indien », Études rurales, 194 (2) : 11-46.

Burguet D., 2014, Figures des maîtres rituels. Les devins-guérisseurs dans l'histoire et aujourd'hui : savoir, action et pouvoir à Madagascar, Thèse de doctorat, Paris, EHESS. 
CAPone S., 1999, La Quête de l'Afrique dans le candomblé. Pouvoir et tradition au Brésil, Paris, Karthala.

— (DIR.), 2004, dossier thématique « Religions transnationales », Civilisations, 51 (1-2).

— 2010, «Religions “en migration" : de l'étude des migrations internationales à l'approche transnationale », Autrepart, 56 (4) : 235-259.

CApone S. \& Salzbrunn M., 2018, «À l'écoute des transnationalisations religieuses. Introduction », Civilisations, $67: 11-21$.

DEJEAN F. \& ENDELSTEIN L., 2013, « Approches spatiales du religieux : jalons épistémologiques et orientations contemporaines ", Carnets de géographes, 6, <http://journals.openedition.org/cdg/908>; <DOI : https://doi.org/10.4000/cdg.908>.

Fancello S. \& Mary A. (DIR.), 2010, Chrétiens africains en Europe. Prophétismes, pentecôtismes et politique des nations, Paris, Karthala.

FiélouX M. \& LOMBARD J., 1995, « Du royaume à la ville : le territoire des possédés », in J.-F. VINCENT, D. DORY \& R. Verdier (dir.), La construction religieuse du territoire, Paris, L'Harmattan : 323-337.

Fourchard L., MARY A. \& OTAYEK R., 2005, Entreprises religieuses transnationales en Afrique de l'Ouest, Paris, Karthala.

Guedu P., 2009, « Africain, Akan, Panafricain et Afro-Américain », Civilisations, 58 (1): 73-92.

- 2011, Panafricanisme, religion akan et dynamiques identitaires aux États-Unis. Le chemin du Sankofa, Paris, L'Harmattan.

LAMBeK M., 2002, The Weight of the Past, Living with History in Mahajanga, Madagascar, New York, Palgrave Macmillan («Contemporary Anthropology of Religion »).

Laurent P.-J. \& Plaideau C., 2010, «Esprits sans patrie : une analyse de la "religion du voyage" dans les îles du Cap-Vert », Autrepart, 56 (4) : 39-55.

LEGRIP-RANDRIAMBELO O., 2016, «Le prophète et la souveraine. Utilisation des récits biographiques de figures historiques par les guérisseurs betsileo (Madagascar) », Cahiers de littérature orale, $<$ http://journals.openedition.org/clo/3035>, $<$ DOI : https://doi.org/10.4000/clo.3035>.

LeVITT P., 2007, God Needs no Passport, How Immigrants are changing the American Religious Landscape, New York, The New Press.

Meintel D. \& LeBlanc M.-N., 2003, «Présentation : la mobilité du religieux à l'ère de la globalisation », Anthropologie et Sociétés, 27 (1) : 5-10.

Mourre M., 2020, «Histoire et émotions. La mise en scène de la Maison des esclaves à Gorée au Sénégal », Ethnologie française, 177 (1) : 77-89, <doi:10.3917/ ethn.201.0077>. 
PÉdron-Colombani S., 2010, «Le culte de Maximón. Entre monde maya et migrations internationales ", Autrepart, 4 (56) : 75-92, <https://www.cairn.info/revue-autrepart2010-4-page-75.htm>, < DOI : 10.3917/autr.056.0075>.

PRENCIPE L., 2012, «La religion des migrants en tant qu'élément de cohésion sociale », Migrations Société, 139 (1): 101-120.

TALl E. K., 2002, «Comment se construit et s'invente une tradition religieuse », Cahiers d'Études africaines, XLII (3), 167, <http://journals.openedition.org/etudesafricaines/151>; <DOI : https://doi.org/10.4000/etudesafricaines.151>.

Trouillet P-Y. \& LASSEur M., 2016, « Introduction. Les lieux de culte entre territoires et mobilités du religieux : cadre théorique et perspectives contemporaines depuis les Suds », Les Cahiers d'Outre-Mer, 274 (2) : 5-38.

ZEMPLÉNI A., 1996, «À propos de "La construction religieuse du territoire” », Journal des africanistes, 66 (1-2): 335-340. 